\title{
Minimizing the number of late jobs in a stochastic setting using a chance constraint
}

\author{
Marjan van den Akker · Han Hoogeveen
}

Published online: 21 September 2007

(C) Springer Science+Business Media, LLC 2007

\begin{abstract}
We consider the single-machine scheduling problem of minimizing the number of late jobs. We omit here one of the standard assumptions in scheduling theory, which is that the processing times are deterministic. In this scheduling environment, the completion times will be stochastic variables as well. Instead of looking at the expected number of on time jobs, we present a new model to deal with the stochastic completion times, which is based on using a chance constraint to define whether a job is on time or late: a job is on time if the probability that it is completed by the deterministic due date is at least equal to a certain given minimum success probability. We have studied this problem for four classes of stochastic processing times. The jobs in the first three classes have processing times that follow: (i) A gamma distribution with shape parameter $p_{j}$ and scale parameter $\beta$, where $\beta$ is common to all jobs; (ii) A negative binomial distribution with parameters $p_{j}$ and $r$, where $r$ is the same for each job; (iii) A normal distribution with parameters $p_{j}$ and $\sigma_{j}^{2}$. The jobs in the fourth class have equally disturbed processing times, that is, the processing times consist of a deterministic part and a random component that is independently, identically distributed for each job. We show that the first two cases have a common characteristic that makes it possible to solve these problems in $O(n \log n)$ time through the algorithm by Moore and Hodgson. To analyze the third and fourth problem we need the additional assump-
\end{abstract}

Supported by EC Contract IST-1999-14186 (Project alcom-FT).

M. van den Akker $(\bowtie) \cdot$ H. Hoogeveen

Department of Computer Science, Utrecht University, P.O Box

80089, 3508 TB Utrecht, The Netherlands

e-mail:marjan@cs.uu.nl

H. Hoogeveen

e-mail: slam@cs.uu.nl tion that the due dates and the minimum success probabilities are agreeable. We show that under this assumption the third problem is $\mathcal{N} \mathcal{P}$-hard in the ordinary sense, whereas the fourth problem is solvable by Moore and Hodgson's algorithm.

We further indicate how the problem of maximizing the expected number of on time jobs (with respect to the standard definition) can be tackled if we add the constraint that the on time jobs are sequenced in a given order and when we require that the probability that a job is on time amounts to at least some given lower bound.

Keywords Scheduling · Sequencing · Single machine . Number of late jobs $\cdot$ Stochastic processing times . Minimum success probability . Chance constraint . Dynamic programming $\cdot$ NP-hardness

\section{Introduction}

We consider the following single-machine scheduling problem. The machine is assumed to be continuously available from time zero onwards, and it can perform at most one job at a time. The machine has to execute $n$ jobs, denoted by $J_{1}, \ldots, J_{n}$. Performing job $J_{j}$ requires a period of length $p_{j}$, and the execution of this job is preferably finished by its due date $d_{j}$. If job $J_{j}$ is finished after its due date, then it is marked as late. The objective is to minimize the number of late jobs. Since it does not matter at which time a late job is finished, such a job can just as well be skipped altogether; the machine then only carries out the jobs that will finish on time. Hence, the problem then 'changes' to selecting the set of jobs that are executed by the machine, under the constraint that each selected job must be completed by its due date. We assume that all jobs are available at time zero. We 
want to do the selection process at time zero, such that a rejected job can look for another machine to be executed by.

This is one of the classical problems from scheduling theory. Moore (1968) has shown that this problem is solvable in $O(n \log n)$ time by an algorithm that since then is known as Moore-Hodgson's algorithm. In this setting, each job is equally important. In many applications, however, some jobs are more important than others. This importance can be measured by assigning a positive weight $w_{j}$ to each job $J_{j}$ $(j=1, \ldots, n)$; the objective function then becomes to minimize the total weight of the late jobs. Lawler and Moore (1969) show that this problem is solvable in $O\left(n \sum p_{j}\right)$ time by dynamic programming. Karp (1972) shows that pseudopolynomial running time is unavoidable for this problem (unless $\mathcal{P}=\mathcal{N} \mathcal{P}$ ) by establishing $\mathcal{N} \mathcal{P}$-hardness in the ordinary sense, even if all due dates are equal.

We consider this problem in a stochastic setting: we assume that the processing times are not deterministic but stochastic variables. We consider four specific classes of instances. In the first one the processing times are stochastic variables that are distributed according to a gamma distribution with parameters $p_{j}$ (which varies per job) and $\beta$ (which is equal for all jobs). The gamma distribution is often applied to model the processing time of a job (see for instance Law and Kelton 2000). The second class of processing times is used to model a production process where items are produced that work well with probability $r$ and malfunction with probability $(1-r)$; a job $J_{j}$ corresponds then to an order of $p_{j}$ correctly functioning items. The corresponding processing time then follows a negative binomial distribution with parameters $p_{j}$ and $r$. In the third class the processing times consist of a deterministic component $p_{j}$ and a random disturbance, which we assume to be identically distributed for each job. This can be used to model the situation that the disturbances in the production process are not job-related but due to some side-equipment that is used by each job in the same way. In the last case, we assume that the processing times follow a normal distribution with known expected value $p_{j}$ and known variance $\sigma_{j}^{2}$.

We suppose that each due date is deterministic, which is reasonable, as they are specified by the customer issuing the request. More importantly, we further assume that this customer is willing to accept a delayed completion of his/her order, if the company can convince him/her that the planning is such that the probability that the order is delayed is 'small enough'. This is achieved by guaranteeing that the probability that the order is on time is at least equal to some given lower bound value, which we define as the minimum success probability, and which we denote by $y_{j}(j=1, \ldots, n)$. If the customer prefers to be convinced by hard cash, then you can agree that he/her will be compensated if the completion is delayed; when the probability distribution of the completion time of job $J_{j}$ is known, then working with a minimum success probability boils down to specifying an upper bound for the expected compensation payment, which corresponds to a lower bound on the expected profit, and vice versa.

The remainder of the paper is organized as follows. In Sect. 2 we review the problem of minimizing the number of late jobs with deterministic processing times, and we explain Moore-Hodgson's algorithm as a dynamic programming algorithm. We developed this approach ourselves and only found out recently that it has been described in an unpublished paper by Lawler (unpublished) for the more complicated case with agreeable release dates $r_{j}(j=1, \ldots, n)$, where 'agreeable' in this setting implies that the jobs can be numbered such that $r_{i} \leq r_{j}$ implies that $d_{i} \leq d_{j}$, for all $i, j=1, \ldots, n$ (see van den Akker and Hoogeveen 2004). In Sect. 3 we analyze four classes of stochastic processing times. We first prove a general result by specifying a number of constraints such that any problem with stochastic processing times following a probability distribution satisfying these constraints can be reformulated as a deterministic problem, which is solvable in $O(n \log n)$ time, irrespective of the minimum success probabilities; the first and second class of processing times satisfy these constraints. For the other two classes of processing times, we need the additional assumption that the minimum success probabilities and the due dates are agreeable, which here implies that the jobs can be numbered such that $i<j$ implies that $d_{i} \leq d_{j}$ and $y_{i} \geq y_{j}$. We develop dynamic programming algorithms that minimize the (weighted) number of late jobs for these instances; these algorithms are based on the insight gained in Sect. 2. We further discuss the problems that we face in case of general minimum success probabilities. In Sect. 4, we show that the problem with stochastic processing times that follow a normal distribution is fundamentally more difficult than the problem with deterministic processing times by establishing ordinary $\mathcal{N} \mathcal{P}$-hardness for the problem of minimizing the number of late jobs. In Sect. 5, we address a special case of the problem of minimizing the expected number of late jobs under the standard definition. Finally, we draw some conclusions in Sect. 6.

\section{Moore-Hodgson's algorithm reviewed}

In this section, we take a closer look at the problem with deterministic processing times; we will use this insight in Sect. 3. We start with some simple, well-known observations. As mentioned before, the completion time of job $J_{j}$, which we denote by $C_{j}$, has become unimportant once it is greater than the due date. Hence, the late jobs are executed after all the on time jobs (if the late jobs are executed at all). The second observation is that the jobs that are marked as on time are executed in order of nondecreasing due date, which is known as the EDD-order; this is due to Jackson 
(1955), who showed that executing the jobs in EDD-order minimizes the maximum lateness, where the lateness of a job $j$ is defined as $C_{j}-d_{j}$ (this maximum lateness is at most equal to zero if all jobs are on time). Hence, instead of specifying the whole schedule, we can limit ourselves to specifying the set $E$ (for early) containing the on time jobs, as it can be checked whether the jobs in $E$ can be all on time together by putting them in EDD-order. A set $E$ of jobs that are selected to be on time is called feasible if none of them is late when executed in EDD-order. The goal is therefore to find a feasible set $E$ of maximum cardinality. From now on, we will use the following notation. By $|Q|$ and $p(Q)$ we denote the number and total processing time of the jobs in a given set $Q$, respectively. Since the EDD-order is crucial in the design of the algorithm, we assume from now on that the jobs are numbered such that

$d_{1} \leq d_{2} \leq \cdots \leq d_{n}$.

We want to show that Moore-Hodgson's algorithm is in fact a dynamic programming algorithm with a special structure; we need this in Sect. 3. For this dynamic programming algorithm, the following dominance rule is crucial.

Dominance Rule 2.1 Let $E^{1}$ and $E^{2}$ be two feasible subsets of the jobs $\left\{J_{1}, \ldots, J_{j}\right\}$ with $\left|E^{1}\right|=\left|E^{2}\right|$. If $p\left(E^{1}\right)<p\left(E^{2}\right)$, then any solution $E$ with $E \cap\left\{J_{1}, \ldots, J_{j}\right\}=E^{2}$ can be ignored.

Proof Let $E$ correspond to an optimal solution, and suppose that $E \cap\left\{J_{1}, \ldots, J_{j}\right\}=E^{2}$. We will show that replacing the jobs in $E \cap E^{2}$ by the jobs in $E^{1}$ yields a feasible subset $\bar{E}$ of $\left\{J_{1}, \ldots, J_{n}\right\}$; since $|\bar{E}|=|E|$, the subset $\bar{E}$ must then correspond to an optimal solution, too.

To show that $\bar{E}$ is a feasible subset, we must show that in the EDD-schedule of $\bar{E}$ all jobs are on time. Let $\sigma$ and $\pi$ denote the EDD-schedule of the jobs in $\bar{E}$ and $E$, respectively. Due to the numbering of the jobs, we know that the jobs in $E^{1}$ precede the remaining jobs of $\bar{E}$ in $\sigma$; as $E^{1}$ is a feasible subset, these jobs are on time in $\sigma$. The remaining jobs in $\bar{E}$ start $p\left(E^{2}\right)-p\left(E^{1}\right)>0$ time units earlier in $\sigma$ than in $\pi$, and hence these jobs are on time as well.

As a consequence of the dominance rule, the only feasible subsets of $\left\{J_{1}, \ldots, J_{j}\right\}$ with cardinality $k$ that we have to care about are the ones with minimum total processing time. We define $z_{j}(j=1, \ldots, n)$ as the maximum number of jobs in the set $\left\{J_{1}, \ldots, J_{j}\right\}$ that can be on time together; the value of $z_{j}$ will be determined through the algorithm. We further define $E_{j}^{*}(k)\left(j=1, \ldots, n ; k=0, \ldots, z_{j}\right)$ as a feasible subset containing exactly $k$ jobs from $\left\{J_{1}, \ldots, J_{j}\right\}$ with minimum total processing time. We derive a dynamic programming algorithm to solve the problem of minimizing the number of late jobs as follows. We add the jobs one by one in EDD-order. For each combination $(j, k)$, where $j$ $(j=1, \ldots, n)$ refers to the number of jobs that have been considered and $k\left(k=0, \ldots, z_{j}\right)$ denotes the number of on time jobs, we introduce a state-variable $f_{j}(k)$ with value equal to $p\left(E_{j}^{*}(k)\right)$. As an initialization, we define $z_{0}=0$ and put $f_{j}(k)=0$ if $j=k=0$ and $f_{j}(k)=\infty$, otherwise. Suppose that we have determined the values $z_{j}$ and $f_{j}(k)$ for a given $j$ and all $k=0, \ldots, z_{j}$. We first determine $z_{j+1}$ by checking whether job $J_{j+1}$ can be on time given that it succeeds the jobs in $E_{j}^{*}\left(z_{j}\right)$. Hence, we get the recurrence relation

$z_{j+1}= \begin{cases}z_{j}+1 & \text { if } f_{j}\left(z_{j}\right)+p_{j+1} \leq d_{j+1}, \\ z_{j} & \text { otherwise }\end{cases}$

We put $f_{j+1}(0)=0$ and determine $f_{j+1}(k)(k=1, \ldots$, $\left.z_{j+1}\right)$ through the recurrence relation

$f_{j+1}(k)=\min \left\{f_{j}(k), f_{j}(k-1)+p_{j+1}\right\}$.

Here the first term in the minimand corresponds to the option of leaving $J_{j}$ out of the early set, whereas the second term refers to the option of including $J_{j}$. Note that $f\left(j, z_{j+1}\right)=\infty$ if $z_{j+1}=z_{j}+1$. We can compute the values $f_{j}(k)$ in $O\left(n^{2}\right)$ time altogether, from which we immediately determine the minimum number of late jobs as $\left(n-z_{n}\right)$, whereas the optimum schedule can be determined through backtracking.

We will show that by looking at the dynamic programming algorithm in the right way we can obtain MooreHodgson's algorithm. Here we need the close relation between the sets $E_{j}^{*}(k)$ and $E_{j}^{*}(k-1)$ expressed in the following lemma.

Lemma 2.2 A set $E_{j}^{*}(k-1)$ is obtained from the set $E_{j}^{*}(k)$ by removing a job from $E_{j}^{*}(k)$ with largest processing time.

Proof Suppose that the lemma does not hold for some combination of $j$ and $k$. Let $J_{i}$ be a job in $E_{j}^{*}(k)$ with maximum processing time. According to our assumption, $p\left(E_{j}^{*}(k-1)\right)<p\left(E_{j}^{*}(k)\right)-p_{i}$. Determine $J_{q}$ as the job with maximum due date that belongs to $E_{j}^{*}(k)$ but not to $E_{j}^{*}(k-1)$. Now consider the subset $\bar{E}_{j}^{*}(k)=E_{j}^{*}(k-1) \cup$ $\left\{J_{q}\right\}$; we will prove that this subset is feasible, which contradicts the optimality of $E_{j}^{*}(k)$, since $p\left(E_{j}^{*}(k)\right)>p\left(E_{j}^{*}(k-\right.$ $1))+p_{i} \geq p\left(E_{j}^{*}(k-1)\right)+p_{q}=p\left(\bar{E}_{j}^{*}(k)\right)$. We have to check the EDD-schedule for the jobs in $\bar{E}_{j}^{*}(k)$, which is obtained from the EDD-schedule for the jobs in $E_{j}^{*}(k-1)$ by inserting $J_{q}$ at the correct spot. Due to the feasibility of $E_{j}^{*}(k-1)$, the only jobs that may be late are $J_{q}$ and the jobs after $J_{q}$. Observe that the choice of $J_{q}$ implies that from $J_{q}$ onwards the EDD-schedule of $\bar{E}_{j}^{*}(k)$ consists of exactly the same jobs as the EDD-schedule of $E_{j}^{*}(k)$, but 
they are started $p\left(E_{j}^{*}(k)\right)-p\left(\bar{E}_{j}^{*}(k)\right)>0$ time units earlier now. Since $E_{j}^{*}(k)$ is a feasible subset, the same holds for $\bar{E}_{j}^{*}(k)$.

As a consequence, if we know the $\operatorname{set} E_{j}^{*}\left(z_{j}\right)$ (and not just its total processing time), then $f_{j}\left(z_{j}\right)$ is equal to $p\left(E_{j}^{*}\left(z_{j}\right)\right)$ and we can compute $f_{j}\left(z_{j}-1\right)$ as the total processing time of $E_{j}^{*}\left(z_{j}\right)$ minus the processing time of the longest job in $E_{j}^{*}\left(z_{j}\right)$. Plugging this in our recurrence relation (2), then we get

- If $p\left(E_{j}^{*}\left(z_{j}\right)\right)+p_{j+1} \leq d_{j+1}$, then $E_{j+1}^{*}\left(z_{j+1}\right) \leftarrow$ $E_{j}^{*}\left(z_{j}\right) \cup\left\{J_{j+1}\right\}$.

- If $p\left(E_{j}^{*}\left(z_{j}\right)\right)+p_{j+1}>d_{j+1}$, then find the longest job in $E_{j}^{*}\left(z_{j}\right) \cup\left\{J_{j+1}\right\}$ and set $E_{j+1}^{*}\left(z_{j+1}\right)$ equal to $E_{j}^{*}\left(z_{j}\right) \cup$ $\left\{J_{j+1}\right\}$ minus this job.

But this leads exactly to Moore-Hodgson's algorithm, which is defined as follows:

\section{MOORE-Hodgson}

STEP 1. Set $\sigma$ equal to the EDD-schedule for all jobs $J_{1}, \ldots, J_{n}$.

STEP 2. If each job in $\sigma$ is on time, then stop.

STEP 3. Find the first job in $\sigma$ that is late; let this be job $J_{j}$. Find the largest job from the set containing $J_{j}$ and all its predecessors in $\sigma$ and remove it from $\sigma$. Remove the idle time from the resulting schedule by shifting jobs forward; call this new schedule $\sigma$, and go to STEP 2 .

Hence, the Moore-Hodgson's algorithm can be regarded as a dynamic programming algorithm that works with statevariables $g_{j}\left(z_{j}\right)$ only, where the state-variable $g_{j}\left(z_{j}\right)$ then contains the subset of the jobs that lead to $f_{j}\left(z_{j}\right)$. Since the number of state-variables is $O(n)$, it can be implemented to run in $O(n \log n)$ time, whereas our dynamic programming algorithm requires $O\left(n^{2}\right)$ time.

Note that our dynamic programming algorithm immediately carries over to the weighted case, since the dominance rule holds true (with total weight of the on time jobs instead of the cardinality of the set of on time jobs). The statevariables $f_{j}(k)$ are defined as before, but $k$ then assumes the values $0,1, \ldots, \sum_{i=1}^{j} w_{i}$. Hence, the running time of the dynamic programming algorithm becomes $O\left(n \sum w_{i}\right)$. Note that we have to change the recurrence relation to

$$
f_{j+1}(k)=\min \left\{f_{j}(k), f_{j}\left(k-w_{j+1}\right)+p_{j+1}\right\} .
$$

\section{Stochastic processing times}

From now on we assume that the processing times are stochastic variables, which we denote by $\pi_{j}(j=1, \ldots, n)$; we stick to deterministic due dates. Since the completion times are stochastic variables then, we work with a sequence of accepted jobs instead of a schedule with fixed completion times. We further must change the definition of a job being on time, since otherwise we would have to run the schedule first and conclude whether it met its due date afterwards. This definition is based on a chance constraint.

Definition 3.1 Given the sequence in which the jobs are to be executed, a job is considered to be on time if the probability that it is completed by its due date is at least equal to some given probability, which we call the minimum success probability. We call this version of on time stochastically on time.

The objective function is then to minimize the number of late jobs. We solve this problem at time zero and dismiss all nonselected jobs; any rejected costumer will have time to look for an alternative. This approach differs from the wellknown maximization of the expected number of on time jobs. This model has the disadvantage that any job which positively contributes to the expected value will remain in the planning, until it has become impossible to meet the due date; for instance, for the gamma-distribution, in which the processing times can get arbitrarily close to zero, a job will only be removed from the planning at the due date. This objection can be partly overcome by working with a deadline on the starting time, as suggested by Dean (2005). We will come back to this problem in Sect. 5 .

We will consider four classes of instances of stochastic processing times; in all cases we assume that the corresponding stochastic variables are independent. We further assume that the job-specific parameters are integral. The four classes are defined as follows:

- The processing time $\pi_{j}$ of job $J_{j}(j=1, \ldots, n)$ follows a gamma distribution with shape parameter $p_{j}$ and scale parameter $\beta$, which is equal for all jobs. The expected processing time is then equal to $p_{j} \beta$. Since the scale parameter $\beta$ is equal for all jobs, the total processing time $\sum_{i \in S} \pi_{i}$ follows a gamma distribution with parameters $\sum_{i \in S} p_{i}$ and $\beta$.

- The processing time $\pi_{j}$ of job $J_{j}(j=1, \ldots, n)$ is distributed according to a negative binomial distribution with parameters $p_{j}$ and $r$, which is equal for all jobs. The negative binomial distribution can be used to model the process of picking $p_{j}$ correctly functioning items, where each item has a probability of $r$ to function well. The expected number of items that have to be produced such that exactly $p_{j}$ ones are okay is then equal to $p_{j} / r$. As $r$ is equal for each job, the total processing time $\sum_{i \in S} \pi_{i}$ follows a negative binomial distribution with parameters $\sum_{i \in S} p_{i}$ and $r$.

- The processing time $\pi_{j}$ of job $J_{j}(j=1, \ldots, n)$ consists of a deterministic part $p_{j}$ and a random disturbance, where the disturbances are independently, identically distributed random variables. 
- The processing time $\pi_{j}$ of job $J_{j}(j=1, \ldots, n)$ follows a normal distribution with known expected value $p_{j}$ and known variance $\sigma_{j}^{2}$. Hence, $\sum_{i \in S} \pi_{i}$ follows a normal distribution with expected value $\sum_{i \in S} p_{i}$ and variance $\sum_{i \in S} \sigma_{i}^{2}$.

We will show in Sect. 3.1 that the first two classes in fact boil down to deterministic problems. The other two classes require a more intricate approach, where we use the insight gained in the previous section to solve these problems through dynamic programming; this is described in the Sects. 3.2 and 3.3.

\subsection{Gamma and negative binomial distributed processing times}

In this subsection we show that these two distributions satisfy the properties of the theorem below, which implies that these problems can be solved in $O(n \log n)$ time irrespective of the minimum success probabilities. Before stating the theorem, we need some notation. Given an order of the jobs, the completion time of some job $J_{j}$ is equal to the total processing time of $J_{j}$ and all its predecessors, which set we denote by $Q_{j}$ (this set includes $J_{j}$ ).

Theorem 3.2 A class of instances of the problem of maximizing the number of stochastically on time jobs is solvable in $O(n \log n)$ time if the following conditions are satisfied:

- The processing times are independently distributed stochastic variables that are described by one job-specific parameter $p_{j}$.

- The probability distribution is additive in the job-specific parameter, that is, the sum of the processing times of the jobs $J_{i}$ and $J_{j}$ follows the probability distribution with parameter $p_{i}+p_{j}$.

- $P\left[C_{j} \leq d_{j}\right] \geq y_{j}$ does not increase when $p\left(Q_{j}\right)$ increases, irrespective of $y_{j}$.

Proof Because of the first two properties, we know that $C_{j}=\sum_{i \in Q_{j}} \pi_{i}$ follows a distribution that is fully described by $p\left(Q_{j}\right)$ and possibly some common parameters. Given the value of $p\left(Q_{j}\right)$ we can compute $P\left[C_{j} \leq d_{j}\right]$, and because of the third property, we can compute the maximum value of $p\left(Q_{j}\right)$ for which $P\left[C_{j} \leq d_{j}\right] \geq y_{j}$, for example by applying binary search. We use $D_{j}$ to denote this maximum value.

We construct an instance of the deterministic version of the problem of maximizing the number of on time jobs, where each job $J_{j}(j=1, \ldots, n)$ has processing time $p_{j}$ and due date $D_{j}$. Then any feasible solution to this deterministic problem corresponds to a feasible solution of the stochastic problem of equal value and vice versa, since

$p\left(Q_{j}\right) \leq D_{j} \Longleftrightarrow P\left[C_{j} \leq d_{j}\right] \geq y_{j}$
This problem is solved in $O(n \log n)$ time, assuming that we can find all $D_{j}$ values in that time.

Note that we have assumed that the values $D_{j}$ can be determined in $O(n \log n)$ time altogether. Note further that the on time jobs are executed according to the order of the adjusted due dates $D_{j}$, which does not have to coincide with the order of the original due dates $d_{j}$, as the minimum success probabilities do not have to be equal.

Both the gamma distribution and the negative binomial distribution satisfy the properties of Theorem 3.2. The normal distribution is characterized by two parameters, and it cannot be solved in polynomial time, as we show in Sect. 4 . If the expected value and the variance are related, for instance, if for each job the variance is equal to some constant times the expected value, then the problem can be solved in $O(n \log n)$ time again.

A similar approach can be used when we do not work with minimum success probabilities but with some kind of reward function per job: a lower bound on the expected profit then leads to an upper bound $D_{j}$ on $p\left(Q_{j}\right)$, after which the resulting problem of deciding which jobs to carry out can be solved by the algorithm of Moore-Hodgson with deterministic processing times $p_{j}$ and due dates $D_{j}$.

If we look at the weighted problem, where $w_{j}$ is the reward of completing $J_{j}$ on time, then we can follow the same approach if the properties of Theorem 3.2 are satisfied, since the determination of the adjusted due dates $D_{j}$ is independent from the weights. Hence, we can solve the weighted case in time $O\left(n \sum p_{j}\right)$ through the algorithm of Lawler and Moore (1969) or in time $O\left(n \sum w_{j}\right)$ through the dynamic programming algorithm of Sect. 2, given the values $D_{j}(j=1, \ldots, n)$. By applying Karp's reduction (Karp 1972), we can show that the weighted case is $\mathcal{N} \mathcal{P}$-hard in the ordinary sense.

\subsection{Equally disturbed processing times}

Again, we use $C_{j}$ to denote the stochastic completion time of $J_{j}(j=1, \ldots, n)$, and we let $Q_{j}$ denote the set containing all jobs that have been accepted up to and including $J_{j}$. Hence, we have that $C_{j}=\sum_{i \in Q_{j}} \pi_{i}$ follows a distribution that is described by $p\left(Q_{j}\right)$ and the joint disturbance of the jobs in $Q_{j}$. This implies, however, that the second condition of Theorem 3.2 is not satisfied, as the number of jobs in $Q_{j}$, which we denote by $\left|Q_{j}\right|$, is crucial. Hence, we return to the basics of our dynamic programming approach.

The crux behind the dynamic programming algorithm of Sect. 2 was that a fixed order was known in which the jobs could be added without running the risk of missing the optimum, namely the EDD-order based on the $d_{j}$ values. We will show in Theorem 3.3 below that adding the 
jobs in EDD-order, where ties are settled according to nonincreasing $y_{j}$ value, will permit us to find an optimal solution if the minimum success probabilities are agreeable with the due dates, with which we mean that the jobs can be numbered such that $i<j$ implies that $d_{i} \leq d_{j}$ and $y_{i} \geq y_{j}$, for all $i, j=1, \ldots, n$. We assume from now on that the jobs have been renumbered in this way; with we a little abuse of notation, we call this order the EDD-order again.

To compute the probability $P\left[C_{j} \leq d_{j}\right]$ we need to know $p\left(Q_{j}\right)$ and $\left|Q_{j}\right|$. If $\left|Q_{j}\right|$ is given, then $P\left[C_{j} \leq d_{j}\right]$ is maximized by choosing for $Q_{j} \backslash\left\{J_{j}\right\}$ the subset of $\left\{J_{1}, \ldots, J_{j-1}\right\}$ of minimum total deterministic processing time from among the sets with cardinality $\left|Q_{j}\right|-1$ that is feasible; in this respect a subset is feasible if the corresponding jobs can be scheduled such that each job is stochastically on time. Therefore, the required knowledge to compute $P\left[C_{j} \leq d_{j}\right]$ is captured by the information stored in the state-variables $f_{j}(k)$ of the previous section. Hence, we can apply the analysis of the previous section, which leads to MooreHodgson's algorithm. Note that in the algorithm the checking of whether $J_{j+1}$ is on time has to be modified from inspecting ' $f_{j}(k)+p_{j+1} \leq d_{j+1}$ ' to inspecting whether ' $P\left[C_{j+1} \leq d_{j+1}\right] \geq y_{j+1}$ ', that is, we have to check whether ' $P\left[C_{j}+\pi_{j+1} \leq d_{j+1}\right] \geq y_{j+1}$ '. What is left to prove is the validity of the EDD-order.

Theorem 3.3 If $d_{i} \leq d_{j}$ and $y_{i} \geq y_{j}$, then there exists an optimal order for adding the jobs to the dynamic programming algorithm in which $J_{i}$ precedes $J_{j}$.

Proof Consider the subsequence of the jobs corresponding to any optimal solution. If we add the on time jobs in this order to the dynamic programming solution with the tardy jobs interleaved, then the dynamic programming algorithm will come up with this solution or an equivalent one, since our algorithm is guaranteed to find the best solution corresponding to the order in which the jobs are fed into the algorithm. Hence, it suffices to show that there exists an optimum subsequence in which $J_{i}$ precedes $J_{j}$ if both jobs are on time. We prove this by a contradictory argument. If $J_{j}$ precedes $J_{i}$ in this scheduling order, then we modify it by removing $J_{j}$ from its current position and reinserting it immediately after $J_{i}$. Each job affected by this move goes forward in the scheduling order (and hence remains on time), except for $J_{j}$, which gets some more predecessors. The completion time of $J_{j}$ is equally distributed as the completion time of $J_{i}$ in the original scheduling order, as the same subset of jobs is involved, and $J_{i}$ was on time. Since $d_{i} \leq d_{j}$ and $y_{i} \geq y_{j}$, job $J_{j}$ must be on time then, too, which implies that this new scheduling order is at least as good as the previous one.

It is straightforward to solve the weighted case by using state-variables $f_{j}(k, W)$, where $W$ contains the additional information of the total weight of the current set of on time jobs. Note that, in contrast to the deterministic case in Sect. 2, we now need both the total weight of the current set of on time jobs and the cardinality of this set, since knowledge of the cardinality is required for computing $P\left[C_{j} \leq d_{j}\right]$. Hence, the algorithm will then run in $O\left(n^{2} \sum w_{i}\right)$ time. We can use a reduction like the one proposed by Karp to show $\mathcal{N} \mathcal{P}$-hardness of the deterministic problem (which in fact belongs to this class) to verify that pseudo-polynomial running time is the best we can hope for in this case.

\subsection{Normally distributed processing times}

In this subsection we assume that the processing time $\pi_{j}$ of job $J_{j}(j=1, \ldots, n)$ is a normally distributed random variable with expected value $p_{j}$ and variance $\sigma_{j}^{2}$, where the variance is so small that the probability of getting a negative processing time is negligible. We further assume that all $\pi_{j}$ variables are independent of each other. Since the variances can differ per job, this problem is fundamentally more difficult than the previous ones, as we will show in Sect. 4. We have that $C_{j}=\sum_{i \in Q_{j}} \pi_{i}$ follows the normal distribution with mean $\sum_{i \in Q_{j}} p_{i}$ and variance $\sum_{i \in Q_{j}} \sigma_{i}^{2}$. If these two quantities are known, then we can immediately compute the probability $P\left[C_{j} \leq d_{j}\right]$ and compare this to the minimum success probability $y_{j}$. Again, we assume that the due dates and minimum success probabilities are agreeable; Theorem 3.3 shows that there exists an optimal schedule that starts with the on time jobs in EDD-order.

Obviously, not all possible combinations $\left(\sum_{i \in Q_{j}} p_{i}\right.$, $\left.\sum_{i \in Q_{j}} \sigma_{i}^{2}\right)$ are of interest. If we look at all feasible subsets $Q_{j}$ of $\left\{J_{1}, \ldots, J_{j}\right\}$ with equal $\sum_{i \in Q_{j}} \sigma_{i}^{2}$ value and equal cardinality, then our best chance to meet $y_{j}$ is offered by the subset with minimum $\sum_{i \in Q_{j}} p_{i}$ value. On the other hand, if we can choose among all feasible subsets $Q_{j}$ of $\left\{J_{1}, \ldots, J_{j}\right\}$ with equal $\sum_{i \in Q_{j}} p_{i}$ value and equal cardinality, then we would like to have the one with smallest $\sum_{i \in Q_{j}} \sigma_{i}^{2}$ value if $y_{j}>0.5$ and the one with maximum $\sum_{i \in Q_{j}} \sigma_{i}^{2}$ value if $y_{j}<0.5$; if $y_{j}=0.5$, then the variances are irrelevant, which implies that the problem in which all minimum success probabilities are equal to 0.5 can be solved by applying the algorithm by Moore-Hodgson to the instance with deterministic processing times $p_{j}$. Since we may expect that a customer is not satisfied with a probability of less than $50 \%$ that the manufacturer completes his job on time, we assume that $y_{j} \geq 0.5$ for all $j=1, \ldots, n$. Therefore, we work out the dynamic programming algorithm by determining for each value $t \in\left\{0,1, \ldots, \sum_{i=1}^{j} p_{j}\right\}$ and for each cardinality $k=0, \ldots, z_{j}$ the feasible subset $Q_{j}$ with $p\left(Q_{j}\right)=t$ that has minimum total variance, if it exists. The reason behind enumerating the total processing time and minimizing the total variance is that presumably $\sum_{i=1}^{n} \sigma_{i}^{2}>\sum_{i=1}^{n} p_{i}$, which is 
advantageous for the running time of the algorithm. If this is not the case, then we can reverse the role of the variance and the total processing time in the dynamic programming algorithm.

To get the dynamic programming algorithm running, we introduce for each $j=1, \ldots, n ; k=1, \ldots, z_{j}$; and $t=0,1, \ldots, \sum_{i=1}^{j} p_{j}$ the state-variable $f_{j}(k, t)$, which is supposed to denote the minimum variance $\sum_{i \in Q_{j}} \sigma_{i}^{2}$ over all feasible subsets $Q_{j}$ of $\left\{J_{1}, \ldots, J_{j}\right\}$ with $\left|Q_{j}\right|=k$ and $\sum_{i \in Q_{j}} p_{i}=t$. Here $z_{j}$ is defined as the maximum cardinality over all feasible subsets $Q_{j}$ of $\left\{J_{1}, \ldots, J_{j}\right\}$. As our initialization, we put $z_{0}=0$ and define $f_{j}(k, t)=0$ if $j=k=t=0$ and $\infty$, otherwise. As before, we add the jobs in EDD-order (where ties are settled according to nonincreasing $y_{j}$ value). When adding job $J_{j+1}$, we first determine $z_{j+1}$. Thereto, we check for each $f_{j}\left(z_{j}, t\right)$ whether

$$
P\left(z \leq \frac{d_{j+1}-t-p_{j+1}}{\sqrt{f_{j}\left(z_{j}, t\right)+\sigma_{j+1}^{2}}}\right) \geq y_{j},
$$

where $z$ denotes a standard normal variable. If this holds for none of the state-variables $f_{j}\left(z_{j}, t\right)$, then we put $z_{j+1}=z_{j}$; otherwise, we put $z_{j+1}=z_{j}+1$. As the check above indicates, we have to be a bit cautious. The case that $z_{j+1}=$ $z_{j}+1$ does not imply that adding $J_{j+1}$ behind each feasible solution represented by $f_{j}\left(z_{j}, t\right)$ results in $J_{j+1}$ being stochastically on time. If we encounter a situation in which there is no feasible solution corresponding to $f_{j}(k, t)$, then we give it the value $I N F$ (from 'infeasible'). Given the correct values for $f_{j}\left(k^{\prime}, t^{\prime}\right)$, we compute the values $f_{j+1}(k, t)$ as follows. We put $f_{j+1}(0,0)=0$ and compute $f_{j+1}(k, t)$ for $k=1, \ldots, z_{j+1}$ and $t=0, \ldots, \sum_{i=1}^{j+1} p_{i}$ through the following recurrence relation

$$
f_{j+1}(k, t)=\min \left\{f_{j}(k, t), f_{j}\left(k-1, t-p_{j+1}\right)+\sigma_{j+1}^{2}\right\} .
$$

The first term corresponds to including job $J_{j+1}$ in the set of late jobs, whereas job $J_{j+1}$ is included in the on time set in the second case. We work with the special value INF in the following way. If an entry has value $I N F$, then this possibility is ignored by the minimand; if both entries have value $I N F$, then $f_{j+1}(k, t)$ gets the value $I N F$. Furthermore, if the minimum comes from the second term and the first term has value $I N F$, then we check whether $J_{j+1}$ is stochastically on time when $C_{j+1}$ has expected value $t$ and variance $f_{j}\left(k-1, t-p_{j+1}\right)+\sigma_{j+1}^{2}$; if this is not the case, then we set $f_{j+1}(k, t)$ equal to $I N F$ after all. Note that, if the minimum comes from the second term and the first term is not equal to $I N F$, then we know that $J_{j+1}$ will be stochastically on time, since $J_{j}$ was stochastically on time in a comparable but less favorable situation. The optimum value of the objective function is determined as $n-z_{n}$, and the corresponding on time set can be found through backtracking. This algorithm has running time $O\left(n^{2} \sum p_{i}\right)$.

The weighted case can be dealt with by a clone of the above algorithm. In that case, we let $k$ refer to the total weight of the on time jobs instead of the cardinality of the on time set. Hence, the algorithm then runs in $O\left(n \sum p_{i} \sum w_{i}\right)$ time.

\subsection{The nonagreeable case}

Suppose that the due dates and the minimum success probabilities are not agreeable. We start with a two-job example, which belongs to both class three and four, that shows that the EDD-order is not necessarily optimal then. Both jobs have a processing time that follows a normal distribution with $p_{1}=12$ and $p_{2}=8$ and $\sigma_{1}^{2}=\sigma_{2}^{2}=1$. The due dates are $d_{1}=20$ and $d_{2}=21$, and the minimum success probabilities are $y_{1}=0.5$ and $y_{2}=0.95$. A straightforward calculation shows that in the EDD-sequence job $J_{2}$ is late, whereas in the reverse sequence both jobs are on time.

This is bad news of course. On the bright side, for both our special classes it is possible to check in $O\left(n^{2}\right)$ time whether all jobs in a given set can be stochastically on time simultaneously, even if the minimum success probabilities are unequal, through the following rule, which resembles Lawler's Least-cost-last-rule (Lawler 1973): compute the probability distribution of the, yet unknown, last job in the sequence, and determine whether there exists a job that can be sequenced last without violating its minimum success probability. If there is no such job, then this set is not feasible. Otherwise, select any such job, sequence it last, and repeat the procedure for the remaining jobs. The proof is straightforward, and therefore we have omitted it.

Since we are able to check for a given job set whether it is feasible, one approach might be to use the dynamic programming algorithm of the previous subsections, where we use the above algorithm to check whether adding the current job to a feasible subset yields a feasible subset again. Unfortunately, this approach does not work, as follows from the following three-job example: we add job $J_{0}$ to our twojob example with $p_{0}=11, \sigma_{0}^{2}=1, d_{0}=15$, and $y_{0}=0.5$. A quick computation shows that the only possibility of having two jobs on time is to put $J_{2}$ first and $J_{1}$ second. But when we apply our dynamic programming algorithm where we add the jobs in EDD-order, then this solution is eliminated, as the feasible subset of cardinality one from $\left\{J_{0}, J_{1}\right\}$ with minimum processing time contains $J_{0}$ and not $J_{1}$. Note that the dynamic programming algorithm works fine if we add the jobs in the order $J_{0}, J_{2}, J_{1}$.

Therefore we now address the question: in which order should we add the jobs to the dynamic programming algorithm? Finding an optimal one, that is, an order which leads to the optimal solution, is a challenging open question. Below, we give a partial description of an optimal order for 
adding the jobs to the dynamic programming algorithm. If the number of possibly optimal orders is not too big, then one approach is to try them all and pick the best solution.

Theorem 3.4 If $d_{i} \leq d_{j}$ and $y_{i} \geq y_{j}$, where at least one of the inequalities is strict, then we can limit ourselves to orders in which $J_{i}$ precedes $J_{j}$.

Proof The proof follows immediately from the proof of Theorem 3.3.

\section{$4 \mathcal{N} \mathcal{P}$-hardness}

In this section we show that the problem with normally distributed processing times is fundamentally more difficult than the problem with deterministic processing times, which is solved in polynomial time. We show that the problem with equal minimum success probabilities is $\mathcal{N} \mathcal{P}$-hard in the ordinary sense through a reduction from the problem PARTITION, which is known to be $\mathcal{N} \mathcal{P}$-complete in the ordinary sense. PARTITION is defined as follows:

\section{PARTITION}

Given $t$ positive integers $a_{1}, a_{2}, \ldots, a_{t}$ with sum equal to $2 A$, does there exist a subset $Q$ of the index set $\{1, \ldots, t\}$ such that

$\sum_{i \in Q} a_{i}=A ?$

Given any instance of PARTITION, we construct the following instance of the decision variant of our problem. Each $a_{i}$ $(i=1, \ldots, t)$ leads to two jobs: $J_{2 i-1}$ and $J_{2 i}$. Moreover, there is a special job $J_{2 t+1}$. The data are found in Table 1, where the index $i$ runs from 1 to $t$. The symbols $B, M$, and $Z$ stand for numbers. $B$ is such that $B(\sqrt{A+1}-\sqrt{A})>A$; a straightforward computation shows that putting $B$ equal to $2 A \sqrt{A+1}$ rounded down does the trick. The integer $M$ is defined as $A+1+B \sqrt{A}$ rounded down. The integer $Z$ is chosen such that $\sqrt{Z+B^{2} A}-\sqrt{Z}<1$; a straightforward computation shows that $Z=A^{6}(A+1)^{2}$ is sufficient. The minimum success probability $y$ is set equal to 0.8413 ; this choice is motivated by the fact that $P(z \leq 1)=0.8413$ for a standard normal variable $z$.

The decision variant of our problem is defined as the following question: does the instance defined above have a solution in which no more than $t$ jobs are not stochastically on time?

We will show that the answer to PARTITION is 'yes' if and only if the decision problem is answered affirmatively. Before giving a formal proof, we will briefly sketch the outline of the reduction. At most $t$ of the first $2 t$ jobs can be stochastically on time, and we need all of them to get a 'yes' to
Table 1 Data for our instance

\begin{tabular}{llll}
\hline & $p_{j}$ & $\sigma_{j}^{2}$ & $d_{j}$ \\
\hline$J_{2 i-1}$ & $i M$ & $B^{2} a_{i}$ & $\sum_{h=1}^{i} h M+A+B \sqrt{A}$ \\
$J_{2 i}$ & $i M+a_{i}$ & 0 & $\sum_{h=1}^{i} h M+A+B \sqrt{A}$ \\
$J_{2 t+1}$ & $(\mathrm{t}+1) \mathrm{M}$ & $Z$ & $\sum_{h=1}^{t+1} h M+A+\sqrt{Z+B^{2} A}$ \\
\hline
\end{tabular}

the decision variant. Define the index set $Q$ such that $j \in Q$ if and only if $J_{2 j}$ belongs to the set of stochastically on time jobs, for $j=1, \ldots, t$. To let either $J_{2 t-1}$ or $J_{2 t}$ finish on time, we need that $\sum_{j \in Q} a_{j} \geq A$. Then $J_{2 t+1}$ comes into play; the instance is chosen such that $J_{2 t+1}$ can be on time only if $\sum_{j \in Q} a_{j} \leq A$.

Now we come to the formal proof. First suppose that the answer to PARTITION is 'yes'; let $Q$ denote the subset of $\{1, \ldots, t\}$ with $\sum_{j \in Q} a_{j}=A$. Then we construct a 'yes' solution to the decision variant as follows. We construct the set $E$ of on time jobs such that it includes $J_{2 j-1}$ if $j \notin Q$ and $J_{2 j}$ if $j \in Q$, for $(j=1, \ldots, t)$; the last job in $E$ is job $J_{2 t+1}$. Let $J_{[i]}(i=1, \ldots, t+1)$ denote the $i$ 'th job in $E$ when ordered according to the EDD-rule; define $C_{[i]}, p_{[i]}$, and $d_{[i]}$ accordingly. It is readily verified that the expected value of $C_{[i]}$ is no more than $\sum_{h=1}^{i} h M+A$ for $i=1, \ldots, t+1$, and that the variance of $C_{[i]}$ is at most equal to $B^{2} A$, for $i=1, \ldots, t$, whereas $C_{[t+1]}$ has variance $Z+B^{2} A$. Note that the minimum success probability is such that a job $J_{j}$ $(j=1, \ldots, 2 t+1)$ is on time if the expected value of $C_{j}$ plus the standard deviation (which is the square root of the variance) of $C_{j}$ is no more than its due date. Hence, all jobs in $E$ are on time, which implies that no more than $t$ jobs are late, which means 'yes'.

Conversely, suppose that the answer to the decision variant is 'yes'. Let $E$ denote the set of on time jobs. We first show that then exactly one of each pair of jobs $\left\{J_{2 i-1}, J_{2 i}\right\}$ ( $i=1, \ldots, t)$ must belong to $E$. We prove this statement by showing that any set $\bar{E}$ containing $t$ jobs from $J_{1}, \ldots, J_{2 t}$ for which the above does not hold is not feasible. To facilitate the proof, we alter the instance by reducing the processing times and getting rid of the variances; if $\bar{E}$ is not feasible for this instance, then $\bar{E}$ is also infeasible with respect to the original instance. We put $p_{2 i}=i M$ and $\sigma_{2 i-1}^{2}=0$ for $i=1, \ldots, t$; the other data remain unchanged. If we apply Moore-Hodgson's algorithm to this instance, then we find that at most $i(i=1, \ldots, t)$ out of the jobs $J_{1}, \ldots, J_{2 i}$ can be on time, and that the minimum total processing time of such a subset is equal to $\sum_{h=1}^{i} h M$, for $i=1, \ldots, t$. Now we take a closer look at the set $\bar{E}$; we define the sets $\bar{E}_{j}(j=1, \ldots, t)$ as $\bar{E} \cap\left\{J_{1}, \ldots, J_{2 j}\right\}$. We first show that $\bar{E}_{t}$ must contain exactly one job from $\left\{J_{2 t-1}, J_{2 t}\right\}$. Since $\left|\bar{E}_{t}\right|=t$ and at most $t-1$ of the jobs from $J_{1}, \ldots, J_{2 t-2}$ can be on time, at least one of the jobs $J_{2 t-1}, J_{2 t}$ must be- 
long to $\bar{E}_{t}$. If $\bar{E}_{t}$ contains both, then $\bar{E}_{t}$ contains $t-2$ jobs from $\left\{J_{1}, \ldots, J_{2 t-2}\right\}$, and applying Moore-Hodgson shows that the minimum total processing time of such a set is at least $\sum_{h=1}^{t-2} h M+2 t M$. But then

$$
\begin{aligned}
p\left(\bar{E}_{t}\right) & \geq \sum_{h=1}^{t-2} h M+2 t M=\sum_{h=1}^{t} h M+M \\
& >\sum_{h=1}^{t} h M+A+B \sqrt{A}=\max \left\{d_{2 t-1}, d_{2 t}\right\},
\end{aligned}
$$

which shows that such a set $\bar{E}_{t}$ cannot be feasible. Hence, $\bar{E}_{t}$ contains exactly one job from $J_{2 t-1}, J_{2 t}$. We repeat this argument for $\bar{E}_{t-1}$, and find that $\bar{E}_{t-1}$ must contain exactly one job from $J_{2 t-3}, J_{2 t-2}$, etc.

This implies that the set $E$ corresponding to the 'yes' solution must contain exactly one of each pair of jobs $\left\{J_{2 i-1}, J_{2 i}\right\}(i=1, \ldots, t)$; hence, $J_{2 t+1}$ must then also be on time to get the count right. Now that we have established this characteristic of $E$, we return to our original instance. We define $Q$ as the subset of $\{1, \ldots, t\}$ that contains index $i$ if and only if $J_{2 i}$ belongs to the on time set. Let $J_{[t]}$ denote the job from the pair $\left\{J_{2 t-1}, J_{2 t}\right\}$ that is on time. The expected value of $C_{[t]}$ is equal to $\sum_{h=1}^{t} h M+\sum_{j \in Q} a_{j}$, and the variance of $C_{[t]}$ is equal to $B^{2} \sum_{j \notin Q} a_{j}$. If $\sum_{j \in Q} a_{j}<A$, then the variance of $C_{[t]}$ is at least equal to $B^{2}(A+1)$. Hence, the expected value of $C_{[t]}$ plus the standard deviation of $C_{[t]}$ is then at least equal to

$\sum_{h=1}^{t} h M+B \sqrt{A+1}>\sum_{h=1}^{t} h M+A+B \sqrt{A}=d_{[t]}$,

where the ' $>$ ' sign comes from the choice of $B$. This result disqualifies job $J_{[t]}$ as on time, and hence we must have $\sum_{j \in Q} a_{j} \geq A$.

From now on, we use $a(Q)$ as a short-hand notation for $\sum_{j \in Q} a_{j}$. Finally, job $J_{2 t+1}$ comes into play; this job must be on time as well. The expected value of $C_{2 t+1}$ is equal to $\sum_{h=1}^{t+1} h M+a(Q)$, and the variance of $C_{2 t+1}$ is equal to $Z+$ $B^{2}(2 A-a(Q)) \geq Z$. Now suppose that $a(Q)>A$, which implies that $a(Q) \geq A+1$. Hence, the expected value of $C_{2 t+1}$ plus the standard deviation of $C_{2 t+1}$ is at least equal to

$$
\begin{aligned}
& \sum_{h=1}^{t+1} h M+A+1+\sqrt{Z} \\
& \quad>\sum_{h=1}^{t+1} h M+A+1+\sqrt{Z+B^{2} A}-1=d_{2 t+1},
\end{aligned}
$$

where the ' $>$ ' sign comes from the choice of $Z$. But then $J_{2 t+1}$ is late. This contradiction shows that $a(Q)=A$, and we have shown that the answer to PARTITION is 'yes' as well.

Since the decision variant of our problem belongs to $\mathcal{N} \mathcal{P}$, we have proven the following theorem.

Theorem 4.1 The problem of minimizing the number of late jobs on a single machine with normally distributed processing times is $\mathcal{N P}$-hard, even if all minimum success probabilities are equal.

Until so far, we have not worried about the possibility that the actual processing time of a job might become negative. This can virtually be avoided in the above $\mathcal{N P}$-hardness proof by adding to each processing time a huge constant and updating the due dates in a corresponding fashion.

\section{Maximizing the expected number of on time jobs}

In Sect. 3 we introduced the concept of stochastically on time to be able to establish the value of the objective function without waiting until the last job was finished. Another way to circumvent this problem is to use as an objective function the expected number of on time jobs. The quality of a sequence is then measured as

$\sum_{j=1}^{n} P\left(C_{j} \leq d_{j}\right)$,

which expression has to be maximized. If not all jobs are equally important, then we can add weights $w_{j}$ and maximize the total weighted probability.

As mentioned before, a job will always be included in the optimal order as long as there is a positive probability of meeting the due date, no matter how small. To avoid this, we use the minimum success probabilities in this setting, too, and we only choose jobs that are stochastically on time. Hence, we are now looking for a sequence in which each job is stochastically on time and by which the total probability of meeting the due date is maximized. We solve this problem by applying dynamic programming again. Since we consider a job only once in our dynamic programming algorithm, a job that is rejected, although being stochastically on time, does not get a second chance. This approach is correct only if adding this job to the final set of selected jobs cannot lead to a feasible subset, which can be verified by the analogon of Lawler's Least-cost-last-rule (Lawler 1973) formulated in Sect. 3.4.

Note that it may seem optimal to execute the accepted jobs in EDD-order, but this is not always true. Consider the following two-job example. Both jobs have a processing time that follows a normal distribution. The data are 
$p_{1}=12, p_{2}=2, \sigma_{1}^{2}=16$, and $\sigma_{2}^{2}=1$. The due dates are $d_{1}=14$ and $d_{2}=15$, and we choose $y_{1}=y_{2}=0.5$. The expected number of on time jobs is equal to almost 1.29 for the EDD-sequence and 1.5 for the reverse sequence. Note that, if we would have $y_{1}=y_{2}=0.55$, then the reverse sequence would be infeasible, and the EDD-sequence would be optimal. This implies that we can only guarantee that an optimum is found by feeding the jobs to our dynamic programming algorithms in all nondominated orders. Unfortunately, the above example also shows that a job with larger due date and smaller minimum success probability should sometimes precede a job with a smaller due date and a larger minimum success probability, which makes a partial description of the set of optimal orders unlikely. Given that the jobs are added in order of index, where the initial numbering is arbitrary, our algorithms below find the best solution in which the accepted jobs are executed in order of index.

We start with the instance class with processing times that follow a gamma distribution; the class of instances with processing times from a negative binomial distribution can be dealt with in the same way. Given a sequence in which $J_{j}$ is an accepted job, we use $Q_{j}$ to denote the set of accepted jobs up to and including $J_{j}$. To compute $P\left(C_{j} \leq d_{j}\right)$ we only need $p\left(Q_{j}\right)$. To measure the quality of this solution, we need to know $\sum_{i \in Q_{j}} P\left(C_{i} \leq d_{i}\right)$, which we denote as $\operatorname{Pr}\left(Q_{j}\right)$. We can capture this information by using statevariables $f_{j}(t)\left(j=0, \ldots, n ; t=0, \ldots, \sum_{h=1}^{j} p_{h}\right)$, which denote the maximum value $\operatorname{Pr}\left(Q_{j}\right)$ over all subsets $Q_{j}$ of $\left\{J_{1}, \ldots, J_{j}\right\}$ with $p\left(Q_{j}\right)=t$. Working this out is standard, and therefore we omit it for reasons of brevity.

A similar algorithm can be used for the instance class with equally disturbed processing times, but now we need to keep track of $\left|Q_{j}\right|$ as well. We therefore use state-variables $f_{j}(k, t)\left(j=0, \ldots, n ; k=0, \ldots, j ; t=0, \ldots, \sum_{h=1}^{j} p_{h}\right)$, which again denote the maximum value $\operatorname{Pr}\left(Q_{j}\right)$ over all subsets $Q_{j}$ of $\left\{J_{1}, \ldots, J_{j}\right\}$ with $\left|Q_{j}\right|=k$ and $p\left(Q_{j}\right)=t$.

Finally, the instance class with normally distributed processing times can be dealt with by a similar dynamic programming algorithm. We need to keep track of $p\left(Q_{j}\right)$, $\sigma^{2}\left(Q_{j}\right)$, and $\operatorname{Pr}\left(Q_{j}\right)$ in each iteration. Therefore, we use state-variables $f_{j}(s, t)\left(j=0, \ldots, n ; s=0, \ldots, \sum_{h=1}^{j} \sigma_{h}^{2}\right.$; $\left.t=0, \ldots, \sum_{h=1}^{j} p_{h}\right)$, which denote the maximum value $\operatorname{Pr}\left(Q_{j}\right)$ over all subsets $Q_{j}$ of $\left\{J_{1}, \ldots, J_{j}\right\}$ with $\sigma^{2}\left(Q_{j}\right)=s$ and $p\left(Q_{j}\right)=t$.

The weighted versions can be dealt with through straightforward generalizations of the above algorithms.

\section{Concluding remarks}

In this paper, we have looked at the problem of maximizing the (weighted) number of on time jobs in a stochastic envi- ronment. To cope with the stochastic completion times, we have introduced the concept of a job being stochastically on time. This concept is more general than the requirement that the expected completion time is no more than the due date, and it offers the possibility to the client to ask for a higher reliability of on time completion. Moreover, a similar approach can be used in the situation that a penalty has to be paid for tardy completion, which may depend on the actual completion time, and the manufacturer accepts a job only if the expected profit is large enough.

We have analyzed four classes of stochastic processing times, which all allow us to describe the completion times as stochastic variables with an accessible distribution. We have formulated a general theorem that states sufficient conditions that allow us to reformulate the stochastic problem into an equivalent deterministic problem irrespective of the values of the minimum success probabilities. We have further shown that, if only a part of these properties were met, then we can solve the problem given the order in which the accepted jobs are to be executed.

There are several open questions and directions for future research. The first one is to derive more dominance rules that must be satisfied by an optimal order to add the jobs in the dynamic programming algorithm. Another direction is to introduce a 'look-forward' time, during which information can be gathered concerning the actual processing times of the first jobs in the schedule. Closely related to this is to put the problem in an on line context. Finally, it is an interesting question to find out whether there is a link between our model and the model of maximizing the expected number of on time jobs.

We further want to point out that, whereas we have considered the single machine problem only, a similar approach can be used to solve parallel machine problems with stochastic processing times.

Acknowledgements The authors want to express their gratitude to Sem Borst and to an anonymous referee for their helpful comments on an earlier draft of the paper.

\section{References}

Dean, B. C. (2005). Approximation algorithms for stochastic scheduling problems. $\mathrm{PhD}$ thesis, MIT. www.cs.clemson.edu/ bcdean/ bdean_phd_thesis.pdf.

Jackson, J. R. (1955). Scheduling a production line to minimize maximum tardiness (Research Report 43). Management Science Research Project, University of California, Los Angeles.

Karp, R. M. (1972). Reducibility among combinatorial problems. In R. E. Miller \& J. W. Thatcher (Eds.), Complexity of computer computations (pp. 85-103). New York: Plenum.

Law, A. M., \& Kelton, W. D. (2000). Simulation modeling and analysis. New York: McGraw-Hill. 
Lawler, E. L. (unpublished) Scheduling a single machine to minimize the number of late jobs. Unpublished manuscript.

Lawler, E. L., \& Moore, J. M. (1969). A functional equation and its application to resource allocation and sequencing problems. Management Science, 16, 77-84.

Lawler, E. L. (1973). Optimal sequencing of a single machine subject to precedence constraints. Management Science, 19, 544-546.
Moore, J. M. (1968). An $n$ job, one machine sequencing algorithm for minimizing the number of late jobs. Management Science, 15, 102-109.

van den Akker, J. M., \& Hoogeveen, J. A. (2004). Minimizing the number of tardy jobs. In J. Y. -T. Leung (Ed.), Handbook of scheduling, algorithms, models, and performance analysis (pp. 227-243). Boca Raton: CRC Press. 